\title{
Single Molecule Blinking and Photobleaching Separated by Wide-Field Fluorescence Microscopy
}

\author{
Thomas Gensch, $*, \dagger$ Martin Böhmer, ${ }^{*, \dagger}$ and Pedro F. Aramendía ${ }^{*,+}$ \\ Institute for Biological Information Processing, IBI-1, Research Centre Jülich GmbH, 52425 Jülich, Germany, \\ and INQUIMAE, Departamento de Química Inorgánica, Analítica y Química Física, FCEN, Universidad de \\ Buenos Aires, Pabellón 2, Ciudad Universitaria, C1428EHA Buenos Aires, Argentina
}

Received: March 3, 2005; In Final Form: May 20, 2005

\begin{abstract}
Single molecule fluorescence detection of Atto590 in poly(vinyl alcohol) was achieved by using a wide-field epifluorescence microscope with CCD-camera detection. Image sequences are obtained from which the time traces of the detected molecules are built. We find a distinctive difference between the time evolution of the fluorescence originating from the molecules detected in the first image of the sequence compared to the time evolution of the fluorescence of the molecules detected in each image of the sequence. Atto590 shows very long blinking times and photobleaching and photoblinking that are both quadratically dependent on the irradiation power density. Our approach allows kinetic separation of photobleaching from blinking. The possibility of choosing different ensembles of molecules is demonstrated and taken advantage of for this aim. Initially dark molecules or low emitting ones that might be overlooked are important to describe the complete ensemble behavior.
\end{abstract}

\section{Introduction}

Photobleaching and blinking of single molecules have attracted attention since the initial studies in the field. ${ }^{1-11}$ Photobleaching is an irreversible process that is caused by lightinduced chemical reactions, which transform the fluorophore into a molecule with significantly decreased emission probability. In single molecule fluorescence detection, the one-step photobleaching (a so-called on-off step) of the observed molecule is a characteristic difference compared to ensemble observation with an apparent exponential decrease of the emission signal as a function of illumination time. In contrast to photobleaching, blinking is characterized by a reversible decrease or disappearance of the emission taking place in the time scale of microseconds to seconds, which is much longer than the lifetime of the (emitting) excited singlet state (typically $1-10 \mathrm{~ns}$ ). Blinking is most often light induced (i.e., photoblinking). It can be a result of transient population of non- or lowemissive states, such as excited triplet states, ${ }^{1,6,12}$ photoisomers, ${ }^{2,3,6}$ or other reversible metastable species (such as geminate radicals or species originated by proton or electron transfer). $4,9,13$ Processes such as molecular rotation, ${ }^{14-17}$ spectral shift due to local interactions, ${ }^{18}$ or conformational changes ${ }^{18,19}$ that modify the absorption and/or emission properties of the molecule can also cause blinking behavior.

Photobleaching imposes a limit to the useful time of a fluorophore. It is usually an undesirable process of low quantum efficiency for common fluorescent dyes (in the order of $10^{-5}$ to $\left.10^{-8}\right),{ }^{20}$ but it may be a required feature, for example, in acceptor photobleaching for quantification of Förster resonance energy transfer.

Photoblinking can render useful information on the photophysics of the compounds by analyzing the duration and

\footnotetext{
$\dagger$ Research Centre Jülich, GmbH. E-mail: t.gensch@fz-juelich.de (T.G.) m.boehmer@fz-juelich.de (M.B.). Fax: +49-2461-61-4216.

$\doteqdot$ Universidad de Buenos Aires. E-mail: pedro@qi.fcen.uba.ar. Fax + 541145763341 .
}

distribution of on and off times. ${ }^{1}$ From these, the lifetime and population efficiency of the dark states can be obtained. Triplet states are often responsible for microsecond to millisecond blinking. The lifetime of triplet states (i.e., in this case, the duration of the off states of the fluorescent molecule) is dependent on the concentration of molecular oxygen. Photoisomers can be responsible for dark states lasting from milliseconds to seconds, which can have the same consequences as photobleaching for short time measurements. If blinking times are very long, this process may be very difficult to discern from permanent photobleaching. The family of the green fluorescent protein (GFP) displays blinking periods up to several tens of seconds. ${ }^{2,3}$ Recently, very long blinking periods of rhodamine $6 \mathrm{G}$ in poly(vinyl alcohol) (PVA) were demonstrated to arise on a reversible photoproduction of radical species involving a reaction of the dye with the polymer. ${ }^{4}$

Confocal fluorescence microscopy is often used to detect single molecules by fluorescence, ${ }^{21-23}$ where the molecules are usually immobilized on a surface or in a matrix. The fluorescence image is built by moving the diffraction-limited focus of the excitation light over the sample by scanning either the focus or the sample. In this procedure, a bias toward the registration of the most intensely emitting molecules of the ensemble is suspected and often occurs. Furthermore, observation of the molecules as a function of time is only possible for one molecule at a time (single molecule fluorescence time trace) or with a very low temporal resolution on the order of $1-100 \mathrm{~s}$ with long intermittent breaks of the observation. ${ }^{7}$ Wide-field excitation combined with epifluorescent image detection with a CCD camera is a recent development with the advantage of detecting and tracing molecules in high quantities at the same time. ${ }^{24}$

We used a wide-field fluorescence microscopy setup to follow the time evolution of Atto590 in PVA. Atto590 is a rhodamine derivative with very favorable characteristics for single molecule detection. Its structure is shown in Figure 1. It has a high fluorescence quantum yield and photostability. Bleaching and blinking are temporarily quantified and kinetically discerned. 


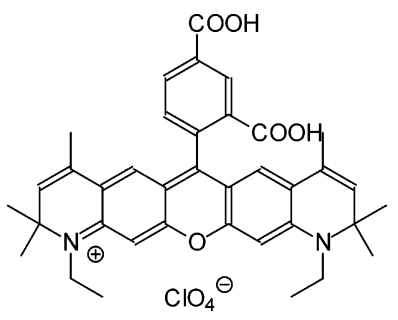

Figure 1. Structure of Atto590.

The possibility of choosing different ensembles of molecules is demonstrated and taken advantage of for this aim. We show that, initially, dark molecules or molecules with low emission intensity, which might be overlooked in the first observation, are important to describe the complete ensemble behavior.

\section{Experimental Section}

The single molecule fluorescence setup is based on an Olympus IX70 inverted microscope with epifluorescent detection. The excitation light source used in this study is the 530 nm line of an $\mathrm{Ar}^{+}-\mathrm{Kr}^{+}$laser (Stabilite $2018 \mathrm{RM}$, Spectra Physics, Mountain View, CA). The light passed through a variable neutral density filter (Laser2000, Germany) and was fed into the microscope through quartz fiber optics and a singleport imaging condenser (Till Photonics, Martinsried, Germany) mounted to the epifluorescence port of the microscope. Here, the laser light from the fiber exit was imaged critically into the observation plane of the microscope by the condenser optics and reflected via a dichroic mirror (DRLP560, Omega Optical, Battleboro, VT) onto the sample using an oil immersion objective (UPlanApo, 60 magnification, 1.4 NA, infinity corrected, $0.170 \mathrm{~mm}$ cover slide corrected, Olympus, Japan). The irradiated spot on the sample is greater than $100 \times 100 \mu \mathrm{m}$. Excitation light was polarized at random on the sample. The emitted light, collected by the objective, was passed through the dichroic mirror and a band-pass filter (590AF60, Omega Optical, Battleboro, VT). Fluorescence was detected with a Peltier-cooled back-illuminated CCD camera (Photometrics CoolSNAP HQ, Roper Scientific, Tucson, AZ). The chip of the CCD has a total active area of $8.98 \times 6.71 \mathrm{~mm}$ divided into $1392 \times 1040$ pixels (size: $6.45 \times 6.45 \mu \mathrm{m}$ ) and was operated at $-30{ }^{\circ} \mathrm{C}$. The CCD was positioned at the intermediate image plane of the side port of the microscope. Fluorescence images were acquired with the software RS Image (Roper Scientific, Tucson, AZ) and further processed and analyzed by programs written in Matlab. Image sequences of up to 50 exposures were stored with exposure times from 0.2 to $4 \mathrm{~s}$ per image according to the irradiation light power density. One pixel represents an area of $108 \times 108 \mathrm{~nm}$ in the sample plane with the used objective. In most cases, due to limitations by storage speed and capability, only one-quarter of the field of view was captured, so the normal image was $696 \times 520$ pixels (i.e., 75 $\times 56 \mu \mathrm{m}$ in the sample). The light power was measured at the exit of the fiber optics with a Coherent Power Meter (Lasercheck, Coherent, Auburn, CA).

Samples were prepared by spin coating dilute dye solutions $\left(10^{-9}-10^{-11} \mathrm{M}\right)$ in water with $1.0 \% \mathrm{w} / \mathrm{w}$ poly(vinyl alcohol) (PVA, 99+\% hydrolyzed, average $M_{\mathrm{w}} 89000-98000$, Aldrich) onto a cover glass (Menzel, Germany, $0.17 \mathrm{~mm}$ thick, $24 \times 60$ $\mathrm{mm})$. The fluorescent dye, Atto590, was obtained from AttoTec (Siegen, Germany).

\section{Results}

Raw images were analyzed using threshold criterion to identify single molecules. To achieve this, the background was processed first. The broad irradiation field has an uneven distribution of photon density. This produces a scattered lightdominated background that is not constant in the image. However, the excitation light intensity differs not more than $20 \%$ in the whole viewing field and less than $10 \%$ in the onequarter portion of the image selected here for observation. The background was fitted to a biquadratic function of the position at the image and subtracted from the total photon counts in each pixel. This resulted in a nearly zero intensity for the background and mean noise amplitude of \pm 10 counts independent of the position, but slightly dependent on the exposure time. The threshold was set to 3 times this noise amplitude; its typical value was 30 counts. Molecules were identified as a cluster of at least five connected pixels above the threshold. The center of gravity of this cluster was calculated, and from the center of this cluster, a square spot, $5 \times 5$ pixel large $(540 \times 540 \mathrm{~nm}$ in the sample), was built to measure the total emission of the molecule. A mask was created including all molecules identified in this way in a given picture. This mask was used to follow the evolution of the emitting molecules in time. This procedure allowed the back and forth tracking of emitting molecules because the image selected to build the mask can be any image of the sequence. In this way, it is possible to identify molecules that have not been observed in the first image because of being in the off state, that is, blinking molecules. With this procedure, one obtains a truly representative population by picking all emitting molecules in the field of view. A bias of the statistics toward the brightest molecules in the first image is prevented, which often occurs in the confocal scanning detection scheme.

In Figure 2, four (out of a time series of 50) fluorescence images are depicted. They show the emitting molecules identified in images 1, 4, 20, and 50 of an image sequence of Atto590 molecules in a PVA matrix. The total number of fluorescent molecules is monotonically decreasing from image 1 to image 50 , as expected due to the phenomenon of photobleaching. The fluorescence in certain fluorescent spots disappears in a single step, as shown for molecules in Figure 2E,F. However, a number of molecules fluorescing in images 4,20 , or 50 are not visible in image 1 (see molecules in Figure $2 \mathrm{G}, \mathrm{H}$ ). This can only be explained by blinking. The on state of some of the molecules, for which the fluorescence intensity traces are shown in Figure $2 \mathrm{E}-\mathrm{H}$, seems to have more than one intensity level. This can be explained by rotation of the molecule or different equilibria between on and slow ( $<$ seconds) off states.

Figure 3 illustrates the different results of the analysis of a single image using masks built from two different images of the sequence. It compares image 20 with molecules identified creating a mask using the threshold and cluster criteria described above in image 20 (Figure 3B, which is identical to Figure 2C), with the same image 20 but now viewed with the mask built from image 1 (Figure 3A). Evidently, those molecules that were off in image 1 but on in image 20 do not appear as bright spots in Figure 3A. Additionally, those bright spots in image 1 that were off in image 20, either due to blinking or bleaching, do not appear either in Figure 3B. In fact, from the 17 molecules detected in Figure 3B, only 10 appear as bright spots in Figure 3A. This means that, at least, seven molecules show blinking behavior and were in the off state when image 1 was taken. Instead of image 20 (used in Figure 3), any of the 50 images can be used to build a mask for analyzing the whole image sequence. The use of these masks provides similar observation probabilities of blinking Atto590 molecules with long off times $(\gg 10 \mathrm{~s}$ ) compared to the nonblinking fraction of the molecules. 

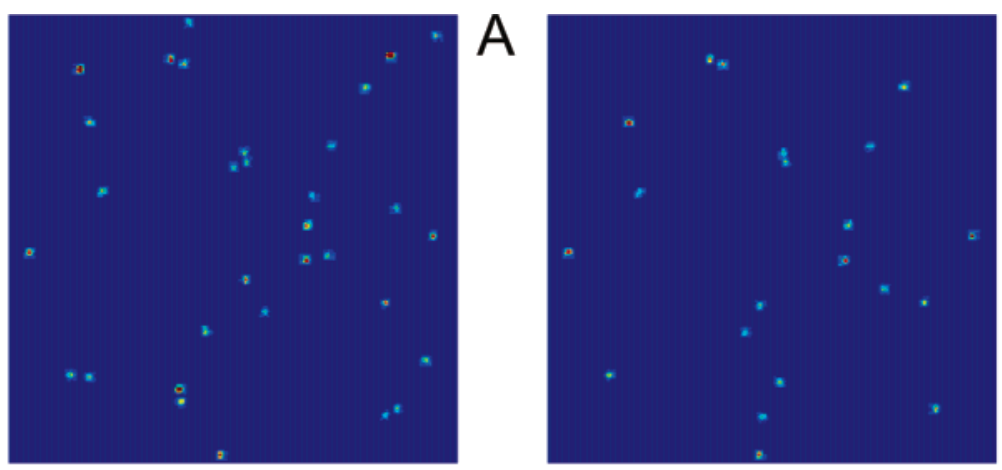

B
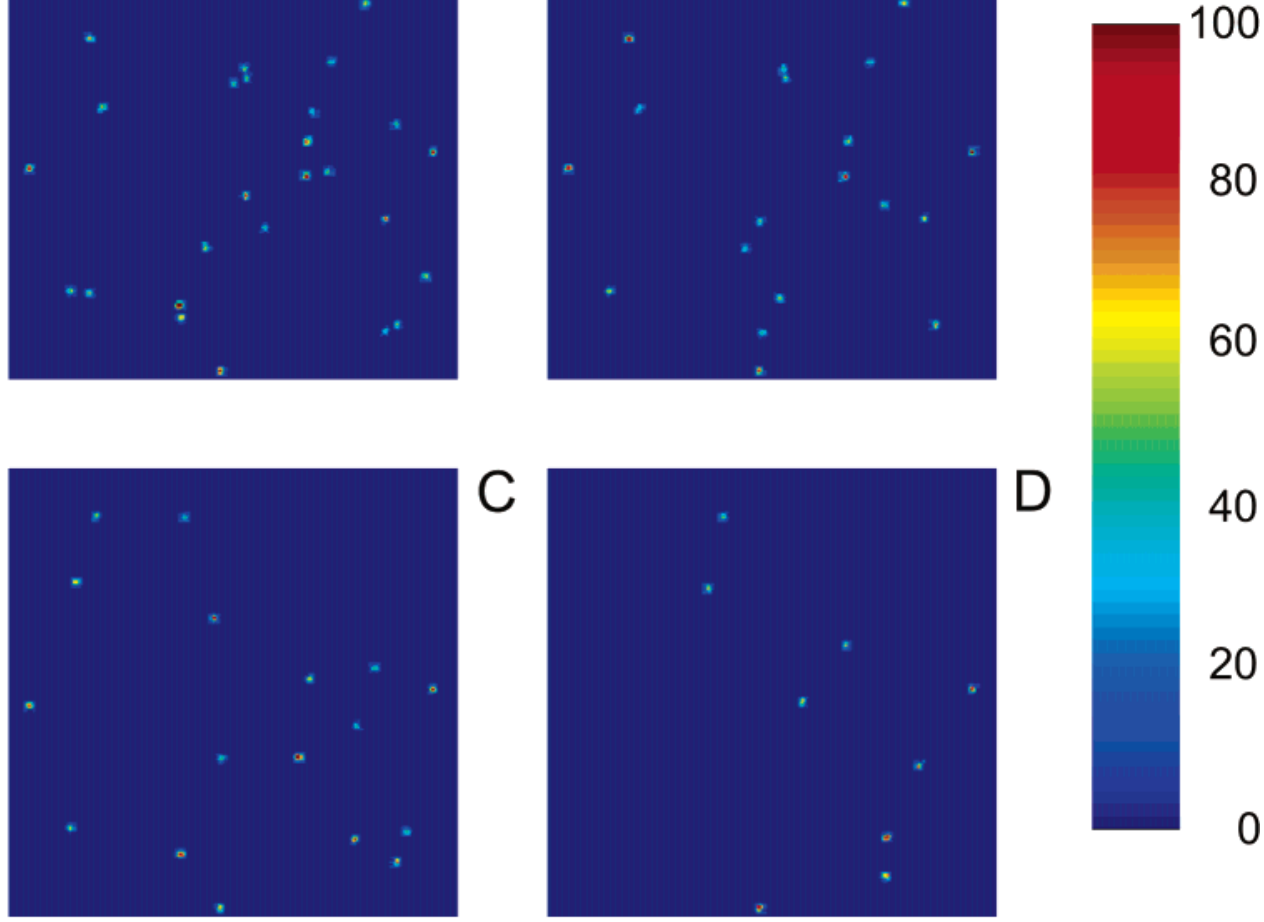

C

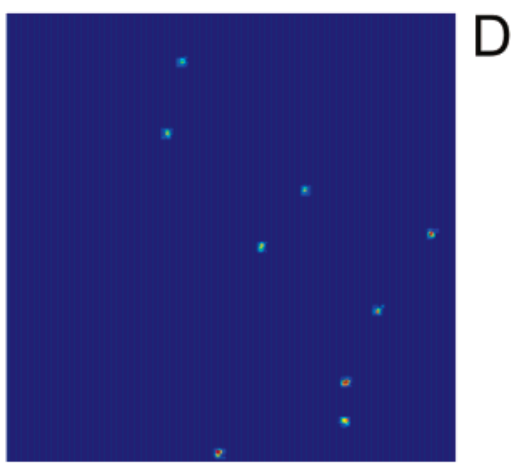

40

20
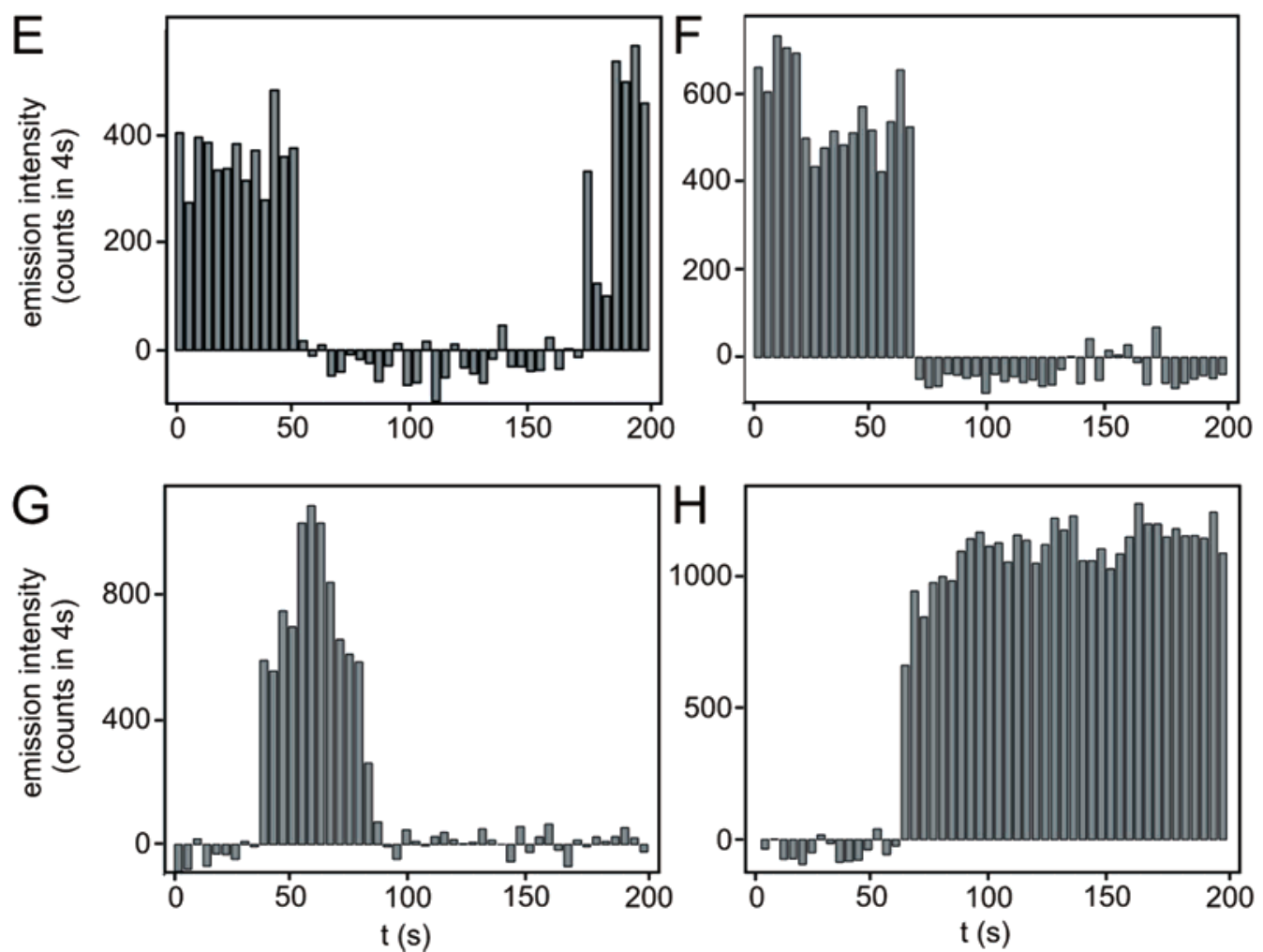

Figure 2. Atto590 molecules in PVA, $4 \mathrm{~s}$ exposure each picture, $11.2 \mathrm{~mW}$ at $530 \mathrm{~nm}$. All pictures show a detail of $200 \times 200$ pixels large $(21.6$ $\times 21.6 \mu \mathrm{m}$ in the sample). Bright spots are $5 \times 5$ pixel areas $(540 \times 540 \mathrm{~nm}$ total area in the sample), where emitting molecules are detected according to the threshold criterion (see text). Note, that different molecules, due to blinking and photobleaching, are detected in the four images, A-D; (A) image 1, (B) image 4, (C) image 20, (D) image 50. The color bar is the number of counts above average background level in each pixel. ( $\mathrm{E}$ and $\mathrm{F})$ : Fluorescence time traces of two molecules identified in image 1. $(\mathrm{G}$ and $\mathrm{H})$ : Fluorescence time traces of two molecules that were dark in image 1 and bright in image 20. In $(\mathrm{E}-\mathrm{H})$, intensity is given as total counts in $4 \mathrm{~s}$ in the $5 \times 5$ pixel area delimiting the molecule.

The positions of the emitting molecules coincide within \pm 1 pixel in a sequence. Diffusion of the molecules out of the spots defined in the mask during the image sequence does not occur. Therefore, an erroneous identification of "apparently blinking molecules" due to diffusion can be excluded.
Figure 3C displays the average intensity of the emitting molecules in ensembles identified by masks built from images $1,4,20$, and 50, respectively. In all four ensembles, the average emission intensity in image 1 is nearly the same. This intensity decreases by $8-15 \%$ in the monitored time interval. The nearly 

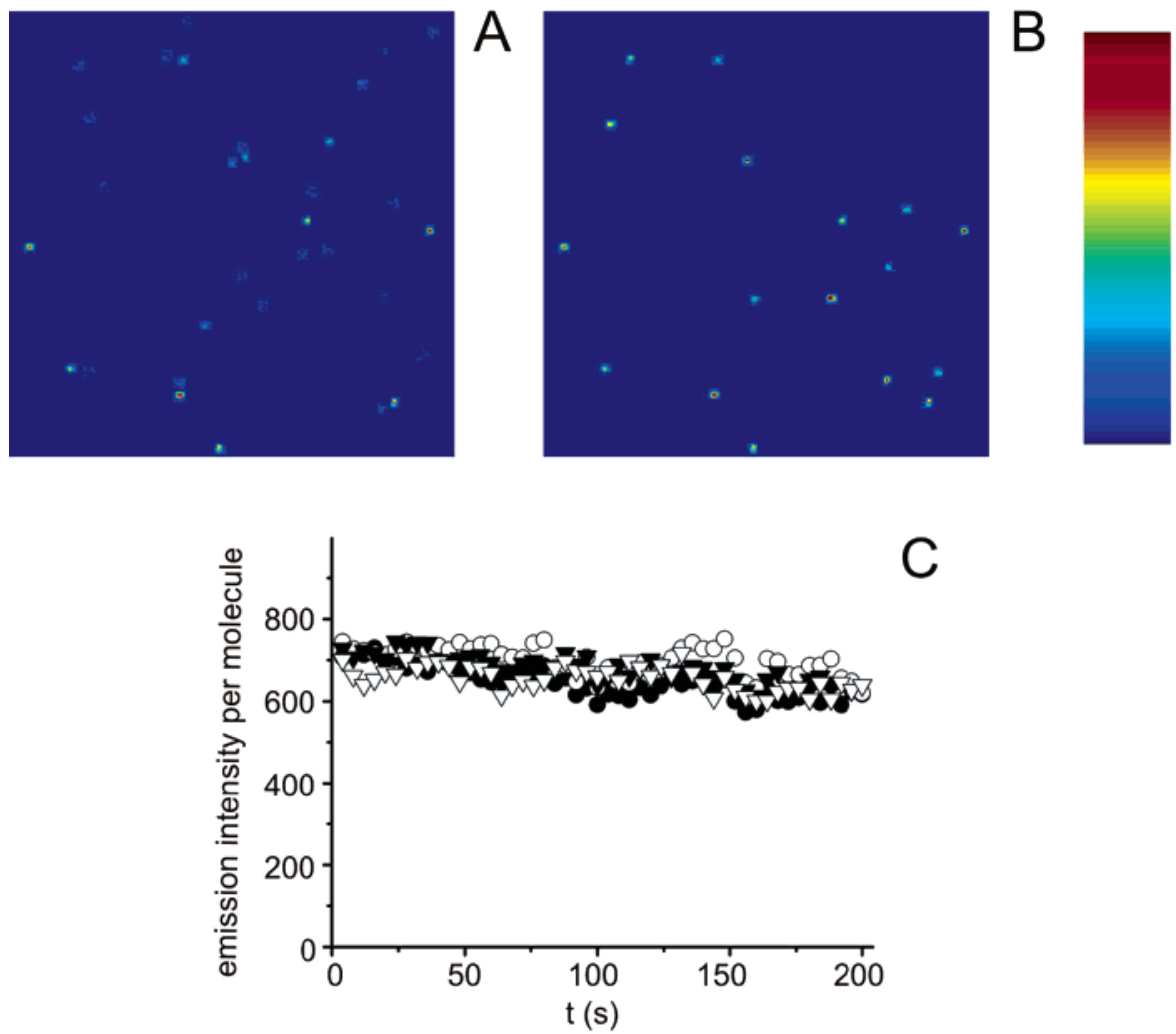

Figure 3. Atto590 molecules in a PVA film coated on a cleaned cover glass. Incident light flux: $11.2 \mathrm{~mW}$ at $530 \mathrm{~nm}$; sequence of 50 images; 4 s exposure per image. (A) Image 20 of the sequence seen through the mask built from image 1. Bright spots correspond to emitting molecules, provided they were emitting in image 1. (B) Same image but showing bright spots identified directly in image 20 according to the same threshold criterion used in image 1 . The color bar gives the number of counts above average background in each pixel. (C) Average fluorescence intensity of emitting molecules, identified by masks built in different images, throughout the 50 images. Full circles, mask built from image 1 ; hollow circles, mask built from image 4; full triangles, mask built from image 20; hollow triangles, mask built from image 50.

TABLE 1: Emission Intensity, on Frame 20, of Different Sets of Molecules Identified on This Frame of the Sequence of Figure 2 (corresponding to Figure 2C)

\begin{tabular}{|c|c|c|c|c|}
\hline $\begin{array}{l}\text { frame } 1 \\
\text { (status) }\end{array}$ & $\begin{array}{l}\text { frame } 4 \\
\text { (status) }\end{array}$ & $\begin{array}{l}\text { frame } 20 \\
\text { (status) }\end{array}$ & $\begin{array}{c}\text { emission intensity } \\
\text { on frame } 20^{a} \\
\text { (No. of molecules) }\end{array}$ & $\begin{array}{c}\text { standard } \\
\text { deviation }^{a}\end{array}$ \\
\hline $\mathrm{ON}$ & & $\mathrm{ON}$ & 747 (77) & 500 \\
\hline \multirow[t]{3}{*}{ OFF } & & $\mathrm{ON}$ & $583(50)$ & 270 \\
\hline & $\mathrm{ON}$ & $\mathrm{ON}$ & $750(79)$ & 492 \\
\hline & $\mathrm{OFF}$ & $\mathrm{ON}$ & $570(48)$ & 271 \\
\hline
\end{tabular}

${ }^{a}$ Values given in counts in $4 \mathrm{~s}$.

identical average intensity in image 1 and the very similar intensity time course of the four curves show that the ensemble of molecules selected on the different frames is very similar to those molecules emitting in image 1 with respect to their photophysical properties (especially their photobleaching behavior). The decrease of the average intensity at longer illumination times can be explained assuming that the brighter molecules will be the most absorbing ones (e.g., due to the orientation of their dipole moment with respect to the image plane) and thus have the highest bleaching rate.

Table 1 summarizes the mean emission intensity of different sets of molecules identified on frame 20. The two sets of molecules that were emitting on both frames 1 and 20 or on both frames 4 and 20 have a significantly higher mean emission intensity (ca. 750 counts in $4 \mathrm{~s}$ ) than those molecules that were emitting on frame 20 and were off either in frame 1 (mean emission of 583) or in frame 4 (mean emission of 570). These two latter sets correspond surely to blinking molecules. The difference of the means of the blinking molecules compared to the nonblinking ones is statistically significant, with a high probability $(\sim 97 \%)$ as obtained from a t-test. Therefore, we can conclude that blinking molecules, while they are emitting, are dimmer than the molecules with lower blinking probability. This can be explained based on the work of Zondervan et al. ${ }^{4}$ They demonstrate that the dark state responsible for the bleaching of rhodamine in PVA is a radical arising probably on electron or hydrogen transfer from the polymer matrix. In any case, the $-\mathrm{OH}$ moiety of PVA is the most chemically reactive part of the polymer, and it is not unreasonable to assume that molecules closer to $-\mathrm{OH}$ moieties will be more reactive. Additionally, it is well-known that $-\mathrm{OH}$ groups act as efficient fluorescence quenchers by vibronic coupling. This can explain the lower average emission intensity of molecules with higher blinking probability. Another possible explanation is that these molecules have also a higher probability of very short blinking periods (for example, by triplet population) that also cause smaller emission intensity.

Figure 4 plots the decay of the number of emitting molecules. Single exponential decays are used as fit functions to assess a characteristic lifetime for the decay process. For most of the curves, there is an "offset" caused by molecules not bleaching and a dynamic equilibrium of molecules reverting from bright to dark and dark to bright states. Deviations from monoexponential decays are treated as biexponentials. The kinetic schemes proposed in the Discussion to analyze the photophysics provide a basis for these behaviors.

Figure 4 plots the number of emitting molecules in three different cases. The upper curve (hollow triangles) represents the number of emitting molecules detected in each image of the sequence according to the threshold criterion. The decay follows a single exponential law with a first-order decay rate 


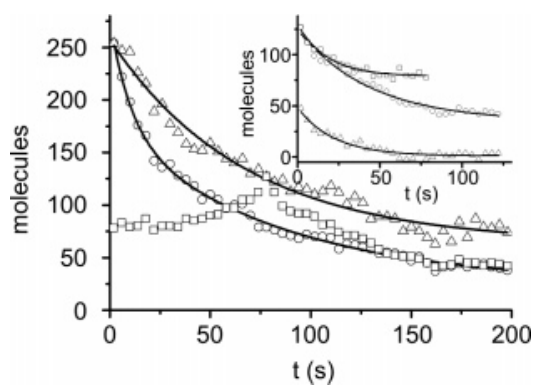

Figure 4. Number of emitting molecules as a function of time for the sample of Atto590 in PVA of Figures 2 and 3. (Main plot) Hollow triangles: number of emitting molecules identified in each picture according to the threshold criterion. Hollow circles: decrease of the number of emitting molecules identified in image 1. Hollow squares: time evolution of the number of emitting molecules identified in image 20. (Inset) Circles: decrease of the number of emitting molecules identified in image 20 for $t>80 \mathrm{~s}$. Squares: time evolution of the number of emitting molecules identified in image 20 for $t<80 \mathrm{~s}$. Triangles: time evolution of the difference between the squares and the circles in the main plot.

constant of $1.5 \times 10^{-2} \mathrm{~s}^{-1}$. The next curve (hollow circles) represents the emitting molecules detected through a mask built from the first image of the sequence. This curve shows the number of emitting molecules as a function of time from those molecules that were emitting at the beginning of the measurement. The decay of the number of emitting molecules in this ensemble follows a biexponential decay law with first-order decay rate constants of $9.8 \times 10^{-2}$ and $1.2 \times 10^{-2} \mathrm{~s}^{-1}$, respectively. Within experimental uncertainty, the lower rate constant of this second curve is equal to the rate constant of the first curve and represents the rate of photobleaching of the ensemble under the experimental conditions. The other decay rate, which is larger than the bleaching rate, is clearly absent from the first curve. It is also clear from Figure 4 that both curves coincide in image 1 , and that afterwards, the total number of fluorescent molecules is always higher than the number of emitting molecules that were also emitting in the first image. This is caused by blinking molecules. In a typical confocal SMS experiment, the photobleaching rate would have been estimated from the molecules identified in image 1 using the mask built in image 1. In fact, the apparent photobleaching rate would be $5.5 \times 10^{-2} \mathrm{~s}^{-1}$, about 5 times larger compared to the real rate $\left(1.2 \times 10^{-2} \mathrm{~s}^{-1}\right)$.

The third curve of Figure 4 (hollow squares) shows the number of emitting molecules in the images when analyzed with the mask built from image 20 . It has a maximum at the time when image 20 was taken. The increase in the number of emitting molecules as the time of the selection is approached is due to the synchronization of the blinking caused by the selection process. From the group of blinking molecules, the more that become fluorescent, the closer the image number is to image 20 , from where the mask was built. The ensemble of molecules emitting in image 20 becomes nonemissive in images taken later than image 20 in the same way as the emitting molecules selected in image 1 (hollow circles), as a consequence of two different processes: blinking and photobleaching. Indeed, the time course of the hollow circles and the hollow squares after image 20 is qualitatively the same. A correct quantitative analysis (biexponential decay) is, however, not possible due to the lower number of contributing molecules and the shorter observation time compared to the time evolution of emitting molecules identified in image 1 .

The inset of Figure 4 shows the kinetic analysis of the third curve of the main plot (represented by hollow squares). There

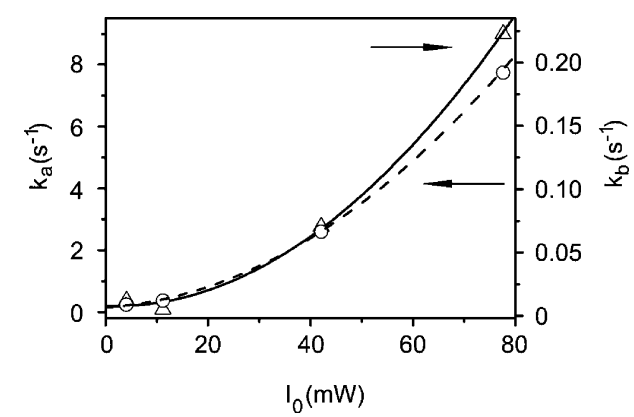

Figure 5. Values of the two first-order decay rate constants of the biexponential fit of the decay of the population identified in the first image for Atto590 samples in PVA for different incident light flux: $k_{\mathrm{a}}$, faster decay rate constant, triangles, experimental points; full line, quadratic fit to the data. $k_{\mathrm{b}}$, slower decay rate constant, circles, experimental points; dashed line, quadratic fit to the data.

are three traces that represent (i) the number of emitting molecules after the selection process (i.e., at $>80 \mathrm{~s}$ (image 20 ; circles)); (ii) the number of emitting molecules before the selection process (i.e., at $<80 \mathrm{~s}$ (image 20; squares)) but inverted on the time axis; (iii) the difference between the trace of hollow squares and of hollow circles in the main plot (upper triangle). The two upper traces, (i) and (ii), of this inset follow a firstorder decay law with rate constants of $2.2 \times 10^{-2}$ and $5.5 \times$ $10^{-2} \mathrm{~s}^{-1}$ for the curves represented by the circles and by the squares, respectively. The trace of upper triangles can also be described by a single-exponential decay function with a rate constant of $4.5 \times 10^{-2} \mathrm{~s}^{-1}$. The decay of the hollow squares trace of the main plot of Figure 4 after $t=80 \mathrm{~s}$ resembles a double exponential function. It should theoretically yield the same results as the hollow circles because the two experiments that they represent are essentially equal but shifted in time. Nevertheless, the fitting of the curve of the hollow squares to a biexponential decay law for $t>80 \mathrm{~s}$ renders ambiguous results due to the limited number of experimental data points available. Instead, it fits well to a single exponential decay with a rate constant intermediate between the two of the hollow circle trace of the main plot. Because of the previously appointed facts, we represent also the difference between the traces of hollow circles and of hollow squares of the main plot. This decay can be fitted to a single exponential with a rate constant similar to the one of trace (ii) of the inset (squares). These latter two curves represent the same phenomenon, namely, the decrease of emitting molecules in the ensemble by blinking and not by photobleaching.

Blinking and photobleaching of Atto590 molecules in PVA were investigated by measuring image sequences using different excitation energies. In each series, the emitting molecules were identified by building a mask in image 1 . The time course of the number of emitting molecules in those ensembles was observed and analyzed with a biexponential model function as in Figure 4 (hollow circle curve). Figure 5 depicts the dependence of the blinking and photobleaching rates on the incident light flux. In this plot, $k_{\mathrm{a}}$ and $k_{\mathrm{b}}$ are the larger and smaller rate constants, respectively. For both rate constants, the dependence can be well described by a quadratic function. This indicates that both processes need the absorption of two photons to take place and points to photoinduced processes from excited states, as already described for other systems under irradiation conditions for single molecule fluorescence experiments. ${ }^{4,10,11}$

\section{Discussion}

We base the discussion of our results on a scheme that takes into consideration five electronic states of Atto590 (Figure 6), 


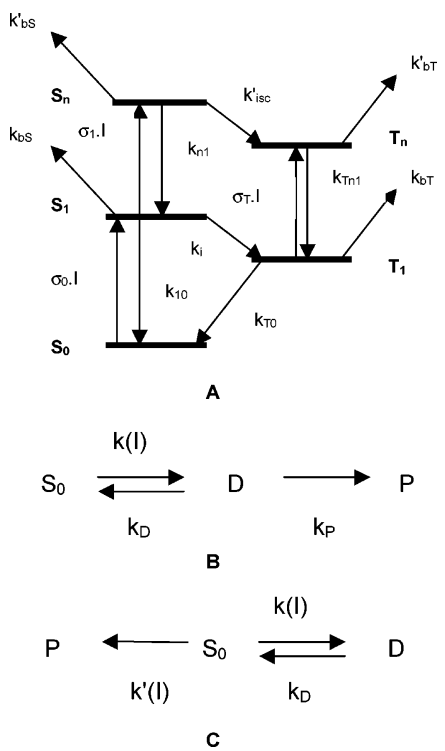

Figure 6. The rate constants $k_{\mathrm{bS}}, k_{\mathrm{bS}}^{\prime}, k_{\mathrm{bT}}$, and $k_{\mathrm{bT}}^{\prime}$ correspond either to processes leading to $\mathrm{D}$ in the sequential mechanism (see Figure 6B) or to the sum of the rate constants leading to $\mathrm{D}\left(k_{\mathrm{DX}}, \mathrm{X}=\mathrm{S}_{1}, \mathrm{~S}_{n}, \mathrm{~T}_{1}\right.$, $\mathrm{T}_{n}$ ) and to $\mathrm{P}\left(k_{\mathrm{PX}}\right)$ in the parallel mechanism (for example, $k_{\mathrm{bS}}=k_{\mathrm{DS} 1}+$ $k_{\mathrm{PS} 1}$, etc.; see Figure 6C), respectively. Under steady-state conditions and low irradiation, $k(I)=a \times I+b \times I^{2}$, and $k^{\prime}(I)=a^{\prime} \times I+b^{\prime} \times$ $I^{2}$. In the parallel and in the sequential mechanism, $k(I)$ and $k^{\prime}(I)$ designate different rate constants, but with the same dependence on the microscopic kinetic parameters.

as used by Eggeling et al. ${ }^{10}$ for the analysis of photobleaching of fluorescent dyes. The five states are: the ground state of the dye $\left(\mathrm{S}_{0}\right)$, an emitting excited singlet state of the dye $\left(\mathrm{S}_{1}\right)$, its corresponding, nonemitting triplet state $\left(\mathrm{T}_{1}\right)$, and two higher excited states, $\mathrm{S}_{n}$ and $\mathrm{T}_{n}$, which must be included to account for the quadratic dependence of blinking and photobleaching on light flux. None of these states can be the off state responsible for the blinking, so a dark state (D) that reversibly returns to the ground state has to be added. Finally, there is a bleached state, $\mathrm{P}$, that represents an irreversibly formed, nonfluorescent photoproduct. The kinetic scheme is represented in Figure 6A. The kinetic equations for the long time blinking originated on $\mathrm{S}_{0}, \mathrm{~S}_{1}, \mathrm{~S}_{n}, \mathrm{~T}_{1}$, and $\mathrm{T}_{n}$ have been solved by Eggeling et al. ${ }^{10}$ They render a rate constant for photobleaching that has a quadratic dependence on the excitation power, in agreement with our observation. Given the time resolution of our experiment, we can assume steady-state conditions for the four electronic excited states $\mathrm{S}_{1}, \mathrm{~S}_{n}, \mathrm{~T}_{1}$, and $\mathrm{T}_{n}$. So, a simplified scheme including three states, namely, $\mathrm{S}_{0}, \mathrm{D}$, and $\mathrm{P}$, is sufficient to model our experimentally determined excitation power dependence of the rate constants for blinking and photobleaching. Two possibilities can be considered, a sequential and a parallel mechanism, respectively, as depicted in Figure 6B and C.

In the sequential mechanism, $\mathrm{D}$ is an intermediate state on the path for permanent bleaching of the dye. The rate constant for the build up of D is quadratically dependent on the light flux, as described by Eggeling et al. ${ }^{10}$ The other two rate constants are first-order thermal processes. Such a scheme has two relaxation rates, referred to as $k_{\mathrm{as}}$ and $k_{\mathrm{bs}}$ :

$$
\begin{gathered}
k_{\mathrm{as}, \mathrm{bs}}=(1 / 2)\left(\sum k \pm \sqrt{\left.\left(\sum k\right)^{2}-4 k(I) k_{\mathrm{P}}\right)}\right. \\
\sum k=k(I)+k_{\mathrm{D}}+k_{\mathrm{P}}
\end{gathered}
$$

In the parallel mechanism, $\mathrm{D}$ and $\mathrm{P}$ are produced by processes involving the absorption of two photons from $\mathrm{S}_{0}$. Both of these rate constants are assumed to have a quadratic dependence on the light flux. The two relaxation rates for this mechanism are:

$$
\begin{gathered}
k_{\text {ap }, \mathrm{bp}}=(1 / 2)\left(\sum k \pm \sqrt{\left.\left(\sum k\right)^{2}-4 k^{\prime}(I) k_{\mathrm{D}}\right)}\right. \\
\sum k=k(I)+k_{\mathrm{D}}+k^{\prime}(I)
\end{gathered}
$$

Our results show that the reversible blinking attains equilibration faster than the decay rate of the permanent photobleaching. This observation allows a further simplification of the expressions for the characteristic rate constants of the sequential and of the parallel mechanism.

$$
\begin{gathered}
k_{\mathrm{as}}=k(I)+k_{\mathrm{D}} \\
k_{\mathrm{bs}}=k_{\mathrm{P}} \frac{k(I)}{k(I)+k_{\mathrm{D}}} \\
k_{\mathrm{ap}}=k(I)+k_{\mathrm{D}} \\
k_{\mathrm{bp}}=k^{\prime}(I) \frac{k_{\mathrm{D}}}{k(I)+k_{\mathrm{D}}}
\end{gathered}
$$

The two mechanisms predict similar dependencies of the rates on the excitation power. Both $k_{\mathrm{as}}$ and $k_{\mathrm{ap}}$ correspond to the equilibration of the reversible blinking and the faster process (corresponding to the plus sign in the full expression of the rates of eqs 1 and 2). As Figure 5 shows, the light-independent part of the faster processes $\left(k_{\mathrm{a}}\right)$ is small. Accordingly, the excitation power dependence of $k_{\mathrm{b}}\left(k_{\mathrm{bs}}\right.$ and $\left.k_{\mathrm{bp}}\right)$, the slower process corresponding to the permanent photobleaching, has a milder dependence on the excitation power. This is evidenced in a more open parabola for $k_{\mathrm{b}}$ in Figure 5.

The fact that the limits of eq 3 cannot be applied strictly to the system may be responsible for the difference between the rate constants obtained for the population that is only blinking, for example, the squares of the inset of Figure 4, and the fast process of the whole ensemble, for example, the upper triangles of the same Figure.

At $4.11 \mathrm{~mW}$ of $530 \mathrm{~nm}$ in $100 \times 100 \mu \mathrm{m}^{2}$, the photon density is $1.1 \times 10^{20}$ photons $/ \mathrm{cm}^{2}$. Considering the absorption crosssection of Atto590 at $530 \mathrm{~nm}, 6.6 \times 10^{-16} \mathrm{~cm}^{2}$, the excitation rate is $7.3 \times 10^{4} \mathrm{~s}^{-1}$ or one photon absorbed every $14 \mu \mathrm{s}$. For the highest light power (the $80 \mathrm{~mW}$ experiment; see Figure 5), the excitation rate is increased to one photon every $700 \mathrm{~ns}$. Only the triplet state, $\mathrm{T}_{1}$, has a lifetime comparable or longer to this average time difference between two absorption events. These considerations point to the triplet state, $\mathrm{T}_{1}$, as the excited state responsible for the absorption of the second photon that ultimately leads to blinking/photobleaching.

In a recent publication ${ }^{25}$ on rhodamine $6 \mathrm{G}$ in PVA, a photobleaching pathway was introduced from the dark state, as well as from the triplet state. The model (a) of this work differs from the sequential model (B) in the addition of a photochemical pathway for the bleaching of $\mathrm{D}$. If $\mathrm{D}$ absorbs the excitation light (and bleaches efficiently), this is a non-negligible pathway since the lifetime of $\mathrm{D}$ greatly exceeds that of $\mathrm{T}_{1}$ and that of $\mathrm{S}_{1}$. Adding the mentioned pathway to the sequential model (B) leads to a modified sequential scheme in which the photochemical step leading from $\mathrm{D}$ to $\mathrm{P}$ is considered with a rate $\Phi_{\mathrm{D} \rightarrow \mathrm{P}} \times \sigma_{\mathrm{D}}$ $\times I[\mathrm{D}]=k_{\mathrm{D} \rightarrow \mathrm{P}}(I)[\mathrm{D}]$. The two relaxation times would be

$$
\begin{gathered}
k_{\mathrm{as}, \mathrm{bs}}=(1 / 2)\left(\sum k \pm \sqrt{\left(\sum k\right)^{2}-4 k(I)\left(k_{\mathrm{P}}+k_{\mathrm{DP}}(I)\right)}\right) \\
\sum k=k(I)+k_{\mathrm{D}}+k_{\mathrm{P}}+k_{\mathrm{DP}}(I)
\end{gathered}
$$


and the functional form of the rate constants on $I$ is not essentially changed.

Physically speaking, the addition of a photochemical pathway leading from $\mathrm{D}$ to $\mathrm{P}$ does not alter the mechanism because it opens a parallel pathway for the photobleaching, which has the same dependence on the incident light flux as the two already considered arising from the excited singlet and triplet states.

The model (b) of Zondervan et al. ${ }^{25}$ is a particular case of the model of Eggeling et al. ${ }^{10}$ It is obtained by eliminating photophysical processes other than intersystem crossing from $\mathrm{S}_{1}$ and by restricting the photobleaching pathways to those arising from $\mathrm{T}_{1}$ and $\mathrm{T}_{n}$. In this case, $\mathrm{T}_{1}$ is the dark state. This cannot be the case in our study because of the very long times of bleaching observed for Atto590 (up to tens of seconds).

Though we are not able to distinguish if the dark state is an intermediate in the bleaching process or not, on the basis of our present data, the procedure outlined here allows one to kinetically distinguish blinking from bleaching, a common question for all fluorescent dyes, and to estimate the proportion of blinking molecules in an ensemble. It can be successfully applied to obtain photophysical information even in relatively complex behavior as the one displayed by Atto590.

Finally, the possibility to analyze the image sequence with different sets of populations obtained from masks built at different observation times lowers the possibility of biased selection of molecules in single molecule spectroscopy.

Acknowledgment. P.F.A. is a member of Carrera del Investigador Científico (CONICET, Argentina) and acknowledges DAAD for a research fellowship and Research Centre Juelich for a travel grant. We thank Francesco Pampaloni and Jörg Enderlein for help in writing the Matlab programs and for helpful discussions. We thank Prof. U. B. Kaupp for his constant support of our work.

Supporting Information Available: Additional data. This material is available free of charge via the Internet at http:// pubs.acs.org.

\section{References and Notes}

(1) Yip, W.-T.; Hu, D.; Vanden Bout, D. A.; Barbara, P. F. J. Phys. Chem. A 1998, 102, 7564.
(2) Peterman, E. J. G.; Brasselet, S.; Moerner, W. E. J. Phys. Chem. A 1999, 103, 10553.

(3) Nifosi, R.; Ferrari, A.; Arcangeli, C.; Tozzini, V.; Pellegrini, V.; Beltram, F. J. Phys. Chem. B 2003, 107, 1679.

(4) Zondervan, R.; Kulzer, F.; Orlinskii, S. B.; Orrit, M. J. Phys. Chem. A 2003, 107, 6770.

(5) Hofkens, J.; Maus, M.; Gensch, T.; Vosch, T.; Cotlet, M.; Kohn, F.; Herrmann, A.; Müllen, K.; De Schryver, F. C. J. Am. Chem. Soc. 2000, $122,9278$.

(6) Köhn, F.; Hofkens, J.; Gronheid, R.; Van der Auweraer, M.; De Schryver, F. C. J. Phys. Chem. A 2002, 106, 4808.

(7) Göhde, W., Jr.; Fischer, U. C.; Fuchs, H.; Tittel, J.; Basché, Th.; Bräuchle, C.; Herrmann, A.; Müllen, K. J. Phys. Chem. A 1998, 102, 9109.

(8) Tinnefeld, P.; Buschmann, V.; Weston, K. D.; Sauer, M. J. Chem. Phys. A 2003, 107, 323.

(9) Liu, R.; Holman, M. W.; Zang, L.; Adams, D. M. J. Phys. Chem. A 2003, 107, 6522.

(10) Eggeling, C.; Widengren, J.; Rigler, R.; Seidel, C. A. M. Anal. Chem. 1998, 70, 2651.

(11) Deschenes, L. A.; Vanden Bout, D. A. Chem. Phys. Lett. 2002, $365,387$.

(12) Hofkens, J.; Schroeyers, W.; Loos, D.; Cotlet, M.; Köhn, F.; Vosch, T.; Maus, M.; Herrmann, A.; Müllen, K.; Gensch, T.; De Schryver, F. C. Spectrochim. Acta A 2000, 57, 2093.

(13) Holman, M. W.; Liu, R.; Adams, D. M. J. Am. Chem. Soc. 2003 , $125,12649$.

(14) Ha, T.; Glass, J.; Enderle, T.; Chemla, D. S.; Weiss, S. Phys. Rev. Lett. 1998, 80, 2093.

(15) Ha, T.; Laurence, T. A.; Chemla, D. S.; Weiss, S. J. Phys. Chem. B 1999, 103, 6839 .

(16) Deschenes, L. A.; Vanden Bout, D. A. J. Phys. Chem. B 2001, 105, 11978.

(17) Deschenes, L. A.; Vanden Bout, D. A. Science 2001, 292, 255.

(18) Hou, Y.; Higgins, D. A. J. Phys. Chem. B 2002, 106, 10306.

(19) Hofkens, J.; Vosch, T.; Maus, M.; Köhn, F.; Cotlet, M.; Weil, T.; Herrmann, A.; Müllen, K.; De Schryver, F. C. Chem. Phys. Lett. 2001, $333,255$.

(20) Schmidt, T.; Kubitscheck, U.; Rohler, D.; Nienhaus, U. Single Mol. 2002, 3, 5.

(21) Ambrose, W. P.; Goodwin, P. M.; Jett, J. H.; Van Orden, A.; Werner, J. H.; Keller, R. A. Chem. Rev. 1999, 99, 2929.

(22) Böhmer, M.; Enderlein, J. Single Molecule Detection on Surfaces with the Confocal Laser Scanning Microscope. In Single Molecule Detection in Solution; Zander, Ch., Enderlein, J., Keller, R. A., Eds.; Wiley-VCH: Berlin, 2002; pp 145-183.

(23) Moerner, W. E. J. Chem. Phys. 2002, 117, 10925.

(24) Blab, G. A.; Oellerich, S.; Schumm, R.; Schmidt, T. Opt. Lett. 2004, $7,727$.

(25) Zondervan, R.; Kulzer, F.; Kol'chenko, M. A.; Orrit, M. J. Phys. Chem. A 2004, 108, 1657. 